J. Lake Sci. (湖泊科学) , 2012, 24(1): 161-168

http: //www. jlakes. org. E-mail : jlakes@niglas.ac.cn

(c) 2012 by Journal of Lake Sciences

\title{
太阳天顶角对太湖恰在水面下辐照度比影响的定量分析
}

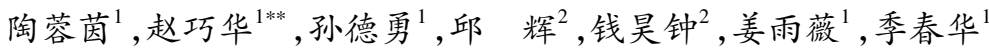 \\ ( 1 : 南京信息工程大学遥感学院,南京 210044) \\ (2:南京信息工程大学大气科学学院,南京 210044)
}

\begin{abstract}
摘 要: 恰在水面下辐照度比变化与吸收系数、散射系数和太阳天顶角存在着密切的关系, 而太阳天顶角对太湖恰在水 面下辐照度比的定量影响程度尚不明确. 为此, 本文基于 2010 年 5 月太湖 27 个样点的水体光学参数和理论数值模拟, 对 太湖水体的情况进行研究. 理论数值模拟结果表明: 当太阳天顶角分别在 $0^{\circ} \sim 25^{\circ}$ 和 $70^{\circ} \sim 89^{\circ}$ 区间内变化时, 对恰在水面下 辐照度比增幅影响较小, 增幅相差不到 $10 \%$; 而当太阳天顶角在 $30^{\circ} \sim 70^{\circ}$ 之间时, 对辐照度比增幅影响很大, 增幅为 $13.13 \% \sim 52.19 \%$. 同时, 利用实测数据对理论结果进行线性相关验证表明, 此数值研究整体上符合太湖的实际情况. 因 此,对水体光学特性以及光对水生生态系统驱动作用研究的时候,应充分考虑太阳天顶角的影响.
\end{abstract}

关键词: 辐照度比; 吸收系数;散射系数;太阳天顶角;太湖

\section{Influence of the solar zenith angle on the irradiance reflectance just beneath the water surface in Lake Taihu}

TAO Rongyin ${ }^{1}$, ZHAO Qiaohua $^{1}$, SUN Deyong $^{1}$, QIU Hui ${ }^{2}$, QIAN Haozhong $^{2}$, JIANG Yuwei $^{1} \&$ JI Chunhua ${ }^{1}$ (1: College of Remote Sensing, Nanjing University of Information Science and Technology, Nanjing 210044, P. R. China) (2: College of Atmospheric Science, Nanjing University of Information Science and Technology, Nanjing 210044, P. R. China)

Abstract: The irradiance reflectance just beneath the water surface is closely related to the absorption coefficient, scattering coefficient and solar zenith angle. However, the effect of solar zenith angle on the irradiance reflectance just beneath the water surface is not clear in Lake Taihu. The influence factors of irradiance reflectance just beneath the water surface in Lake Taihu were analyzed based on the optical parameters and numerical simulates using the data of total 27 sampling sites measured in May, 2010. The results show that while solar zenith angle is in the range of $0^{\circ}-25^{\circ}$ and $70^{\circ}-89^{\circ}$, it has little influence on the growth of irradiance reflectance just beneath the water surface which is less than $10 \%$. While solar zenith angle is in the range of $30^{\circ}-70^{\circ}$, the growth of irradiance reflectance is $13.13 \%-52.19 \%$. The correlation of theoretical results and measured data show that such numerical study is correct for Lake Taihu. Therefore, full consideration should be given to the effect of solar zenith angle when studying on the optical properties of water and the driving mechanism of the light to aquatic ecosystems.

Keywords: Irradiance reflectance; absorption coefficient; scattering coefficient; solar zenith angle; Lake Taihu

水体表观光学特性是指不仅与水体组分有关,而且随外界人射光场变化而变化的水体光学性质,其中 恰在水面下辐照度比 (irradiance reflectance) 就属于表观光学特性参数,定义为紧贴水表下方上行辐照度与 下行辐照度的比值. 它反映了人射到水体中能量的损失, 而这部分损失的能量主要是由水体各组分的吸收 和散射所致, 是分析水质参数理想的表观光学特性之- ${ }^{[1]}$. 同时, 它也是生物光学模型中必不可少的物理 量,携带水体物质组分含量信息 ${ }^{[2]}$,其表征了上行、下行光的综合作用. 同时鉴于光在 $4 \pi$ 立体角的传播特 性,该研究对准确估算水体中生物的光合作用有一定意义. 恰在水面下辐照度比一定程度上反映的是水体 中各组分对光的散射和吸收作用的综合结果，以期探讨太阳天顶角对恰在水面下辐照度比的定量影响程

* 国家自然科学基金项目 (41071070) 和国家水体污染控制与治理科技重大专项项目(2009ZX07101-013)联合资助. $2011-03-07$ 收稿;2011-08-15 收修改稿. 陶蓉茵,女, 1986 年生, 硕士研究生; E-mail : toyoin_004@ 163. com.

** 通信作者;E-mail:qhzhao@ nuist. edu.cn. 
度,及分离各因子对其的影响.

恰在水面下辐照度比与散射系数、吸收系数的关系研究中, 许多学者都通过建立恰在水面下辐照度比 与吸收系数 $(a)$ 、后向散射系数 $(b)$ 的相关模型来讨论它们之间的定量关系. Kirk 等 $^{\left[{ }^{3}\right]}$ 基于实验和水体固有 光学特性推导了辐照度比与吸收系数、后向散射系数之间的关系; 戴永宁等 ${ }^{[1]}$ 利用生物光学模型拟合了巢 湖水表面下方辐照度比 $R\left(0^{-}\right)$与水体散射、吸收系数之间的关系.

恰在水面下辐照度比除了与水体组分的固有光学特性有关, 还与太阳天顶角有关. 基于辐射传输理论, 很多学者开展了恰在水面下辐照度比与太阳天顶角之间关系的研究. Gordon ${ }^{[4]}$ 指出, 体散射函数与太阳天 顶角的变化有关; $\operatorname{Kirk}^{[5]}$ 也通过蒙特卡洛模拟得出水体辐照度比与太阳天顶角的统计关系; Morel 等 $^{[6]}$ 在 I 类水体中建立了太阳天顶角对辐照度比的影响模型, 指出在太阳天顶角小于 $70^{\circ}$ 的条件下, 辐照度比与 太阳天顶角余弦存在线性相关性. 然而关于太阳天顶角对恰在水面下辐照度比定量影响的研究较为 鲜见.

太湖是一个大型浅水湖泊, 其中径流输送的陆源性非藻类颗粒物及有色溶解有机质丰富; 风浪扰动作 用强烈, 易形成底泥的再悬浮, 因而其水体光学特性复杂. 而对恰在水面下辐照度比与太阳天顶角的定量关 系还鲜有研究, 为此, 本文拟通过数值研究的方法寻求太湖恰在水面下辐照度比与太阳天顶角的定量关系, 以便理清固有光学参数及太阳天顶角对恰在水面下辐照度比变化的贡献, 为水生生态环境演变、定量水体 遥感监测的研究奠定基础.

\section{1 材料与方法}

\section{1 研究区站点分布}

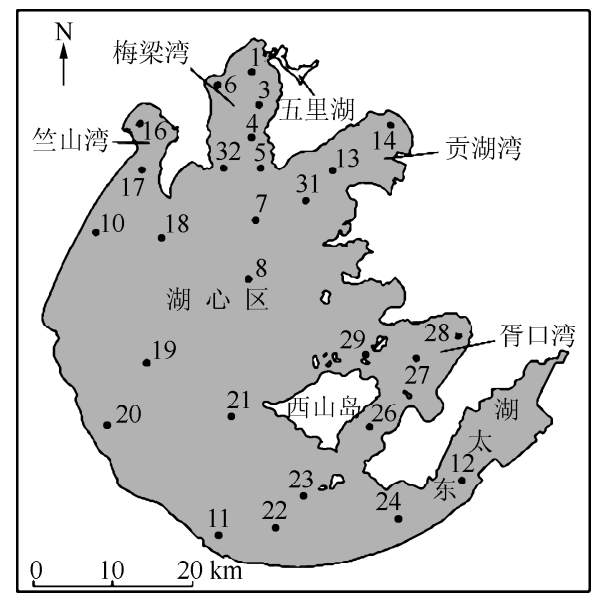

图 1 太湖采样点分布图

Fig. 1 Location of sampling stations in Lake Taihu
2010 年 4 月 29 日 -5 月 2 日在太湖 27 个站 点 (图 1) 测定水体中的上、下行辐照度, 同时采集水 样, 采样点基本覆盖太湖不同生态类型的湖区. 采集 的水样于当天带回实验室分析总悬浮物、叶绿素 $\mathrm{a}$ 和 有色可溶性有机物 (CDOM) 吸收系数. 采样期间天气 均晴好.

\section{2 总悬浮物的光谱吸收系数的测定}

悬浮颗粒物的吸收系数利用定量滤膜技术测

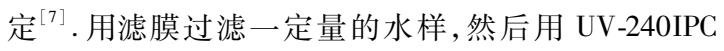
型紫外分光光度计测量过滤所得的滤膜的吸光度, 最 后用吸光度计算吸收系数. 先进行放大因子校正 ${ }^{[8]}$ :

$$
D_{\mathrm{s}}(\lambda)=0.378 D_{\mathrm{f}}(\lambda)+0.523 D_{\mathrm{f}}^{2}(\lambda)
$$

式中, $D_{\mathrm{s}}(\lambda)$ 为校正后滤膜上悬浮颗粒物的吸光度, $D_{\mathrm{f}}(\lambda)$ 为直接在仪器上测定的滤膜上颗粒物的吸光 度减去 $700 \mathrm{~nm}$ 的吸光度, 吸收系数的计算公式为:

$$
a_{\mathrm{p}}(\lambda)=2.303 \frac{S}{V} \times D_{\mathrm{s}}(\lambda)
$$

式中, $a_{\mathrm{p}}(\lambda)$ 为光谱吸收系数, $S$ 为沉积在滤膜上颗粒物的有效面积, $V$ 为被过滤水样的体积.

\section{$1.3 \mathrm{CDOM}$ 光谱吸收系数的测定}

CDOM 的光谱吸收系数测定采用通过孔径 $0.22 \mu \mathrm{m}$ 的滤膜过滤水样提取黄质, 将黄质放人 $1 \mathrm{~cm} \times 4 \mathrm{~cm}$ 的比色血中, 在 UV-2401 PC 型分光光度计 (实测范围 $240 \sim 800 \mathrm{~nm}$ ) 测定 CDOM 的吸光度, 光谱吸收系数的 计算方法为 ${ }^{[9]}$ :

$$
a_{\mathrm{CDOM}}(\lambda)^{\prime}=2.303 \times D(\lambda) / r
$$


计算各波长的吸收系数,再进行散射校正,公式为:

$$
a_{\mathrm{CDOM}}(\lambda)=a_{\mathrm{CDOM}}(\lambda)^{\prime}-a_{\mathrm{CDOM}}(700)^{\prime} \lambda / 700
$$

式中, $D(\lambda)$ 为吸光度, $r$ 为光程路径 $(\mathrm{m}), a_{\mathrm{CDOM}}(\lambda)^{\prime}$ 为波长 $\lambda$ 未校正的吸收系数 $\left(\mathrm{m}^{-1}\right), a_{\mathrm{CDOM}}(\lambda)$ 为波长 $\lambda$ 的吸收系数 $\left(\mathrm{m}^{-1}\right), a_{\mathrm{CDOM}}(700)^{\prime}$ 为参考波段 $700 \mathrm{~nm}$ 处的吸收系数.

\section{4 恰在水面下辐照度比的测量与计算}

上行、下行辐照度使用德国 Trios 公司生产的 SAM-8099 光谱辐射仪测量,其携带三个探头, 分别测定上 行辐亮度、上行辐照度和下行辐照度, 采样波长的范围为 $320 \sim 950 \mathrm{~nm}$, 光谱分辨率为 $3.3 \mathrm{~nm}$. 在每个采样点 测量的水下深度分别为 $0 、 0.1 、 0.2 、 0.3 、 0.4 、 0.5 、 0.6 、 0.7 、 0.8 、 0.9 、 1.0 、 1.1 、 1.2 \mathrm{~m}$, 共 13 个深度. 在进行观 测时, 选择在船体向阳的一面进行, 以避免船体遮挡直射辐射, 且使仪器离开船体一定的距离; 在深度测量 时, 与仪器相连接的绳子上标有相应的刻度, 通过固定在船身的支架将仪器垂直投人水中, 保证测量上行辐 照度的探头向下, 测量下行辐照度的探头向上. 在采集光谱数据的同时,记录采样点的经纬度、时间及风速 风向.

由于水体表面波、船船阴影及仪器自身阴影等,造成太湖中光学参数的测量存在不确定性,但表面波和 船舶等对上行辐照度 $E_{\mathrm{u}}(\lambda, z)$ 、下行辐照度 $E_{\mathrm{d}}(\lambda, z)$ 的影响均随深度增加而减小 ${ }^{[10]}$. 同时由于大型浅水湖 泊风浪作用明显, 可近似看作水体光学均匀, 即认为 $K(\lambda)$ 在观测波长范围内不随深度变化, 可近似地看为 常数. 根据朗伯一比尔定律, 在光学均匀的水体中, 各深度的下行辐照度的计算可表示为 ${ }^{[11]}$ :

$$
E_{\mathrm{d}}(\lambda, z)=E_{\mathrm{d}}(\lambda, 0) \exp \left[-K_{\mathrm{d}}(\lambda) z\right]
$$

式中, $K_{\mathrm{d}}(\lambda)$ 为整个水柱的平均漫射衰减系数, $\lambda$ 为波长, $z$ 为水体表面向下的深度. $E_{\mathrm{d}}(\lambda, z)$ 为深度 $z$ 处波长 为 $\lambda$ 的下行辐照度, $E_{\mathrm{d}}(\lambda, 0)$ 为正好位于水表以下的下行辐照度. $K_{\mathrm{d}}(\lambda)$ 值通过对 $0 \sim 1.2 \mathrm{~m} 13$ 个不同深度 的实测 $E_{\mathrm{d}}(\lambda)$ 值进行指数拟合得到, 回归效果只有当 $R^{2} \geqslant 0.95$, 深度数 $N \geqslant 3$ 时 $K_{\mathrm{d}}(\lambda)$ 值才被接受, 否则视 为无效值. 然后利用拟合得到的 $K_{\mathrm{d}}(\lambda)$, 再次代人公式 $(5)$ 反推得到 $E_{\mathrm{d}}(\lambda, 0) . E_{\mathrm{u}}(\lambda, z)$ 用类似的办法求出, 以消除水体表面波对水下光场的影响.

再利用下式求出实测的太湖恰在水面下的辐照度比 $R\left(\lambda, 0^{-}\right)$:

$$
R\left(\lambda, 0^{-}\right)=E_{\mathrm{u}}(\lambda, 0) / E_{\mathrm{d}}(\lambda, 0)
$$

\section{5 恰在水面下辐照度比的数值计算}

恰在水面下辐照度比 $R\left(0^{-}\right)$依赖于吸收系数 $a$, 散射系数 $b$ 和太阳天顶角 $\theta^{\prime}$. 湖水的衰减系数主要由纯 水、黄色物质、色素和非色素颗粒物的吸收和散射组成. 因此总吸收系数 $a$ 可表示为 $a=a_{\mathrm{CDOM}}+a_{\mathrm{p}}+a_{\mathrm{w}}$, 其 中 $a_{\mathrm{CDOM}}$ 为黄质的吸收系数, $a_{\mathrm{p}}$ 为总悬浮颗粒物的吸收系数, $a_{\mathrm{w}}$ 为纯水的吸收系数. 那么,散射系数 $b$ 则是用 湖水的衰减系数减去吸收系数 $a$ 得到. 根据文献 $[12]$,后向散射概率采取 $\eta=b_{\mathrm{b}} / b=0.019$.

前人研究得出 $R\left(0^{-}\right)$与后向散射系数呈正比,与吸收系数呈反比,存在如下关系 ${ }^{[5]}$ :

$$
R\left(0^{-}\right)=C\left(\mu_{0}\right) b_{\mathrm{b}} / a
$$

式中,光束从天顶角为 $\theta^{\prime}$ 的方向上人射接触到气一水界面, 根据菲涅尔反射定律, 可知将有一部分人射光发 生反射,还有一部分进人水体,恰好在水表层 $0^{-}$处发生折射,此折射角 $\theta$ 的余弦可近似看作为 $\mu_{0}$, 折射系数 取 1.34. 则 $C\left(\mu_{0}\right)$ 是人射光场角分布的函数. 若已知角分布, 则能够求出人射天顶角为 $\theta^{\prime}$ 的 $R\left(0^{-}\right)$. Kirk 在 $\operatorname{Gordon}^{[4]}$ 和 $\mathrm{Kirk}^{[13]}$ 的基础上,通过蒙特卡洛模拟得出, $C\left(\mu_{0}\right)$ 与 $\mu_{0}$ 存在近似的线性关系:

$$
C\left(\mu_{0}\right)=-0.629 \mu_{0}+0.975
$$

代人 (7) 式得到:

$$
R\left(0^{-}\right)=\left(0.975-0.629 \mu_{0}\right) \frac{b_{\mathrm{b}}}{a}=\left(0.975-0.629 \mu_{0}\right) \frac{0.019 b}{a}
$$




\section{2 结果与分析}

\section{1 太湖吸收光谱和散射光谱特征}

在400 650 nm波段内, 由于受悬浮物浓度和 CDOM 浓度影响, 太湖水体吸收系数在短波区间呈 $\mathrm{e}$ 的负 指数形式递减; 在 $676 \mathrm{~nm}$ 附近, 由于浮游植物叶绿素的吸收特征此处出现一个小峰, 峰值的大小取决于水中 色素浓度. 散射系数大致随波长的增加而减小 (图 2), 散射系数与吸收系数的比值在400 580 nm波段内随 着波长的增加而增大, 在 $580 \mathrm{~nm}$ 附近有个波峰, 在 $676 \mathrm{~nm}$ 附近存在一波谷, 主要是由于叶绿素 a 在该处的 吸收峰导致 $b / a$ 值有一个波谷 (图 3). 吸收系数的平均值基本上随着波长增加而减小, 在 $676 \mathrm{~nm}$ 处其平均值 出现一个较小的峰值; 在短波 $440 \mathrm{~nm}$ 处, 各站点变化范围为 $0.81 \sim 5.75 \mathrm{~m}^{-1}$, 在 $580 \mathrm{~nm}$ 处, 吸收系数的变化范 围为 $0.20 \sim 1.10 \mathrm{~m}^{-1}$.
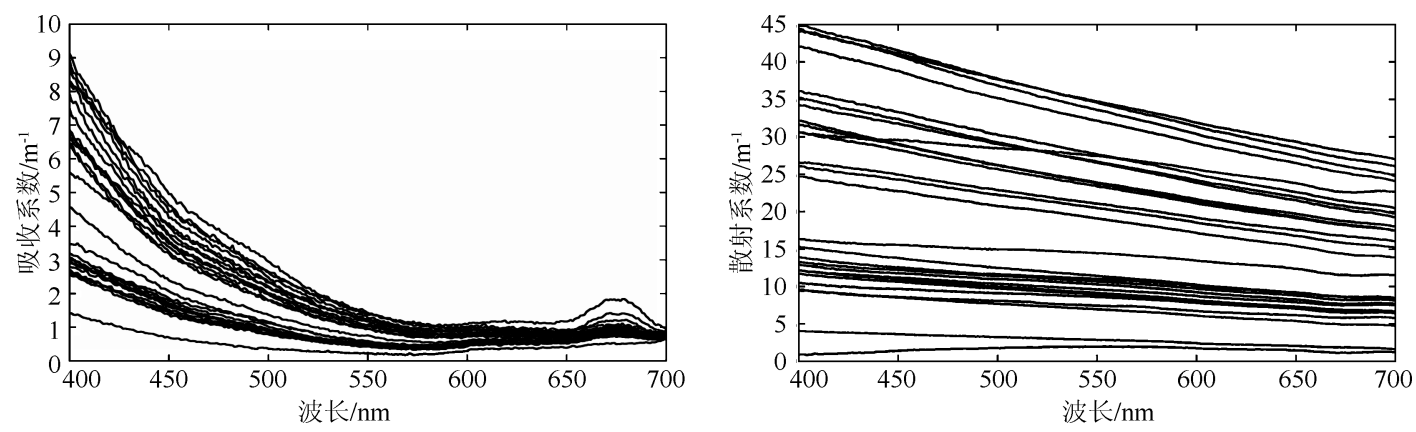

图 2 全湖吸收系数和散射系数的光谱特征

Fig. 2 Spectral characteristics of absorption coefficient and scattering coefficient in Lake Taihu

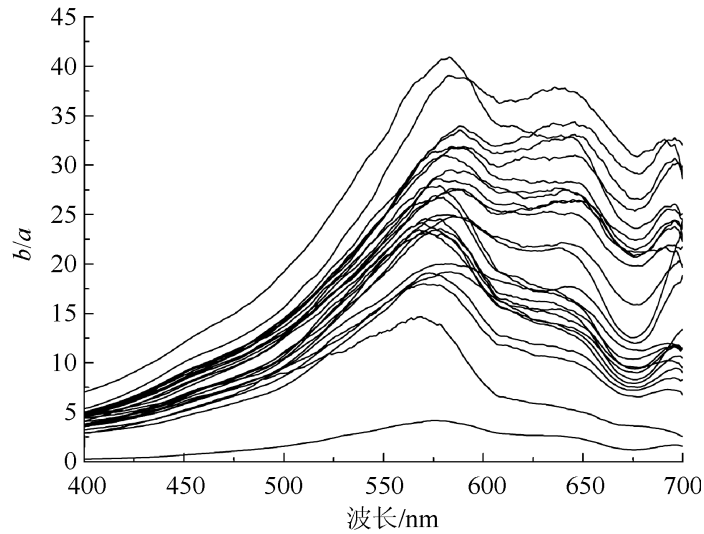

图 3 散射系数与吸收系数之比

Fig. 3 The ratio of the scattering coefficient to the absorption coefficient

各波段吸收系数的标准差从短波到长波依次递 减, 说明各站点吸收系数从短波到长波趋于稳定. 散 射系数的平均值最大值出现在短波处, $440 \mathrm{~nm}$ 处散射 系数的变化范围为 $1.28 \sim 42.17 \mathrm{~m}^{-1}$; 随着波长的增 加, 散射系数平均值逐渐降低, $676 \mathrm{~nm}$ 处的平均值为 $(14.03 \pm 8.12) \mathrm{m}^{-1}$; 各站点标准差较大, 说明站点间 散射系数差异较大. 散射系数光谱形态单一, 基本呈现 随着波长增加而减小的倾斜直线, 并随着波长增加而 逐渐平缓. $b / a$ 的平均值在 $440 \sim 580 \mathrm{~nm}$ 波段间随着波 长增大而增大, $440 \mathrm{~nm}$ 处平均值是 $6.45 \pm 1.90$, $580 \mathrm{~nm}$ 处的平均值是 $25.45 \pm 7.45$; 在 $676 \mathrm{~nm}$ 处有个 波谷, 平均值为 $14.94 \pm 8.39$. 各站点间 $b / a$ 的变化程 度随着波长增加而增大 (表 1 ). 由于各站点水中颗粒 物主导因子类型不同, 悬浮物浓度差异明显, 造成太 湖水体中各参数的显著差异性.

\section{2 理论模拟}

2.2.1 太湖恰在水面下辐照度比与散射系数、吸收系数之比的关系 本研究基于理论公式 (9), 模拟了 $b$ 从 $1 \sim 20 \mathrm{~m}^{-1}$ 变化, 根据后向散射概率取 0.019 , 即后向散射系数 $b_{\mathrm{b}}$ 从 $0.019 \sim 0.38 \mathrm{~m}^{-1}, a=1 \mathrm{~m}^{-1}$, 相应的太阳天顶角 从 $0^{\circ} \sim 89^{\circ}$ 变化 (太阳高度角从 $90^{\circ} \sim 1^{\circ}$ ), 求出不同太阳天顶角下 $R\left(0^{-}\right.$) 的变化趋势.

当太阳天顶角固定时, 恰在水面下辐照度比与 $b / a$ 呈正比 (图 4). 当 $a$ 一定, 光束进人水体, 后向散射的 那部分光成为向上的辐照度, 当后向散射越大时辐照度比越大. 后向散射为固定值时, 水体吸收的越少, 散 射作用比例提高, 上行辐照度增强,因而恰在水面下辐照度比得以提高. 
表 15 个波段吸收系数、散射系数和 $b / a$ 的统计值

Tab. 1 Statistical values of absorption coefficient, scattering coefficient and $b / a$ in five bands

\begin{tabular}{|c|c|c|c|c|c|}
\hline 系数 & 波长/nm & 最小值 & 最大值 & 平均值 & 标准差 \\
\hline \multirow[t]{5}{*}{$a / \mathrm{m}^{-1}$} & 440 & 0.81 & 5.75 & 3.23 & 1.43 \\
\hline & 490 & 0.41 & 3.39 & 1.83 & 0.82 \\
\hline & 550 & 0.24 & 1.43 & 0.84 & 0.37 \\
\hline & 580 & 0.20 & 1.10 & 0.64 & 0.27 \\
\hline & 676 & 0.50 & 1.82 & 0.93 & 0.24 \\
\hline \multirow[t]{5}{*}{$b / \mathrm{m}^{-1}$} & 440 & 1.28 & 42.17 & 21.73 & 12.56 \\
\hline & 490 & 1.69 & 38.50 & 19.95 & 11.39 \\
\hline & 550 & 1.96 & 34.70 & 18.08 & 10.19 \\
\hline & 580 & 1.93 & 33.07 & 17.15 & 9.64 \\
\hline & 676 & 1.13 & 28.05 & 14.03 & 8.12 \\
\hline \multirow[t]{5}{*}{$b / a$} & 440 & 0.54 & 10.61 & 6.45 & 1.90 \\
\hline & 490 & 1.30 & 17.11 & 10.51 & 2.96 \\
\hline & 550 & 3.31 & 31.43 & 20.51 & 5.43 \\
\hline & 580 & 4.30 & 40.64 & 25.45 & 7.45 \\
\hline & 676 & 1.16 & 31.51 & 14.94 & 8.39 \\
\hline
\end{tabular}

结合太湖 5 月份实测的吸收、散射系数 进行恰在水面下的辐照度比与太阳天顶角 的数值研究, 分别选取 $440 、 490 、 550 、 580$ 和 $676 \mathrm{~nm} .12^{\#}$ 点位于东太湖, 悬浮颗粒物和叶 绿素都很少, 辐照度比最小. 而位于梅梁湾的 $32^{\#}$ 点辐照度比一直很高, 梅梁湾的叶绿素、 悬浮物浓度都很大, 散射系数与吸收系数的 比值较大. 在 $676 \mathrm{~nm}$ 波段, $11^{\#} 、 19^{\#} 、 21^{\#} 、 22^{\#}$ 、 $24^{\#} 、 28$ 的辐照度比变的比其他各点大 (图 5). $676 \mathrm{~nm}$ 是叶绿素的敏感波段,这些站点 叶绿素含量很少, 而悬浮物浓度却很高, SS 与 Chl. a 的比值较 $32^{*}$ 点更大, 使得散射作 用大于吸收作用, 其辐照度比就相应增强. 可见, 在太湖水体中, 其辐照度比随着 $b / a$ 值的增大而增大.

\section{2 .2 太湖恰在水面下辐照度比与太阳天}

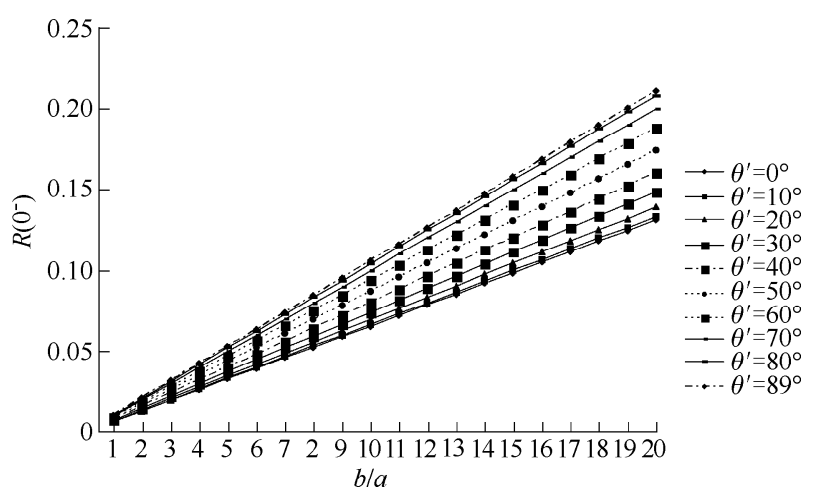

图 4 恰在水面下辐照度比与吸收、散射系数和 太阳天顶角的数值关系

Fig. 4 The relationships between irradiance reflectance just beneath the water surface and the absorption coefficient, scattering coefficient and solar zenith angle

顶角的关系 随着太阳天顶角的减小, 水体辐照度比逐渐减小. 根据水体中颗粒物散射特征, 体散射函数分 布中前向散射远大于后向散射方向. 当太阳天顶角很大时, 由前向散射衍生成的上行辐照度比例增加, 因此 水体恰在水面下辐照度比随着天顶角的增大而增大.

假定太阳天顶角由 $\theta$ 变化到 $\theta^{\prime}$, 则相应的辐照度比由 $R$ 变为 $R^{\prime}$, 则 $R$ 的增幅为:

$$
\frac{R^{\prime}-R}{R}=\frac{C\left(\mu_{0}^{\prime}\right) \frac{\eta b}{a}-C\left(\mu_{0}\right) \frac{\eta b}{a}}{C\left(\mu_{0}\right) \frac{\eta b}{a}}=F\left(\mu_{0}\right)
$$

随着太阳天顶角从 $1^{\circ}$ 增加到 $89^{\circ}$, 辐照度比增大, 其增幅变化趋势是: 天顶角从 $0^{\circ}$ 增加到 $25^{\circ}$ 时, 辐照度 比的增幅为 $1.53 \% \sim 9.27 \%$, 增加趋势平缓, 可以不考虑太阳天顶角变化对辐照度比的影响; 太阳天顶角变 化于 $30^{\circ}$ 与 $70^{\circ}$ 之间时, 辐照度比的增幅为 $13.13 \% \sim 52.19 \%$, 增幅明显, 太阳天顶角变化的影响不容忽略; 当太阳天顶角在 $70^{\circ} \sim 89^{\circ}$ 区间内时, 辐照度比增幅为 $52.19 \% \sim 60.77 \%$, 增幅不明显 (图 6). 在太湖中, 虽然 
各站点的吸收、散射系数不同, 但是其辐照度比增幅的变化趋势基本相同, 即辐照度比增幅变化与太阳天顶 角有很大关系 (图 5 ). 上述变化趋势与折射角 $\theta$ 的余弦函数的变化曲线趋势类似.
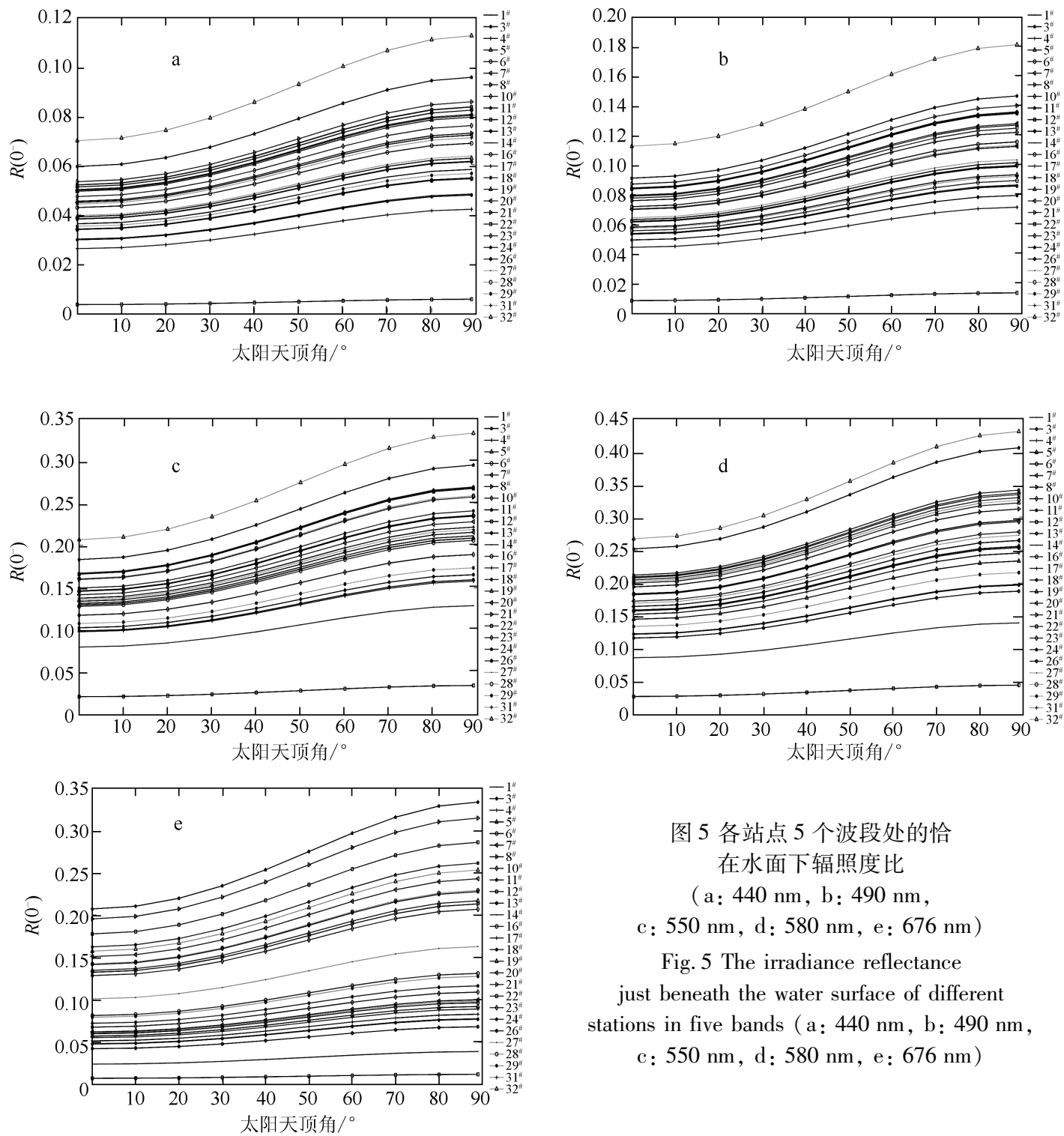

图 5 各站点 5 个波段处的恰 在水面下辐照度比 (a: $440 \mathrm{~nm}, \mathrm{~b}: 490 \mathrm{~nm}$, c: $550 \mathrm{~nm}, \mathrm{~d}: 580 \mathrm{~nm}$, e: $676 \mathrm{~nm}$ )

Fig. 5 The irradiance reflectance just beneath the water surface of different stations in five bands ( a: $440 \mathrm{~nm}, \mathrm{~b}: 490 \mathrm{~nm}$, c: $550 \mathrm{~nm}, \mathrm{~d}: 580 \mathrm{~nm}, \mathrm{e}: 676 \mathrm{~nm}$ )

太阳辐射进人水体传输, 散射的作用并不改变辐射能量大小, 只改变光的传输方向, 而吸收作用则相 反. 当太阳垂直人射时, 光子的行程相对较小, 因而散射作用改变光子传输方向的概率相对较弱, 可见此时 辐照度比基本上由水体的固有光学特性决定; 但随着太阳天顶角逐渐增大, 光传输行程增大, 散射概率提 高, 前向散射一部分成为向上辐照度,造成水体恰在水面下辐照度比随太阳天顶角增大而增大.

\section{3 验证}

以上研究都是基于 (9) 式, 为了验证其可靠性, 以实测太湖区域吸收、散射系数和太阳天顶角为背景场, 采用模拟的恰在水面下辐照度比数据与实测数据进行比较 (图 7). 太阳天顶角是根据上行、下行辐照度测量 时记录的测量时间及采样点的经纬度推算而来, 以保证实测与模拟值太阳天顶角一致. 总体来看, 除了 $6^{\#}$ 点 和 $20^{\#}$ 点差异较大, 27 个站点的实测与模拟的恰在水面下辐照度比趋势一致. 因此, 除去差异最大的 $20^{\#}$ 站 点, 对实测辐照度比和模拟数据进行相关性比较, 结果表明, 模拟值和实测值的相关性较好, 在 $\alpha=0.05$ 的 
显著性水平上, $n=26$, 要求相关系数 $R \geqslant R_{\alpha}=0.38$ (表 2 ). 也就是说, 式 (9) 模拟的恰在水面下辐照度比特 性基本适用于本次数值研究, 其辐照度比与吸收系数呈反比,与后向散射系数呈正比,随着太阳天顶角的增 大而增大. 短波波段模拟结果与实测结果的吻合度不如长波波段好. 各拟合方程的斜率接近于 1 , 截距在 $0.03 \sim 0.06$ 范围内 (表 2), 说明模拟值与实测值之间差异较小, 即模拟效果良好.

表 2 实测与模拟恰在水面下辐照度比的 相关关系 $(n=26)$

Tab. 2 Relationship of measured and simulated irradiance reflectance just beneath the water surface

\begin{tabular}{ccc}
\hline 波长 $/ \mathrm{nm}$ & 拟合方程 & $R$ \\
\hline 440 & $y=1.0979 x+0.0372$ & 0.642 \\
490 & $y=1.2127 x+0.0361$ & 0.726 \\
550 & $y=0.8061 x+0.0623$ & 0.735 \\
580 & $y=1.7028 x+0.0593$ & 0.790 \\
676 & $y=0.906 x+0.0366$ & 0.948 \\
\hline
\end{tabular}

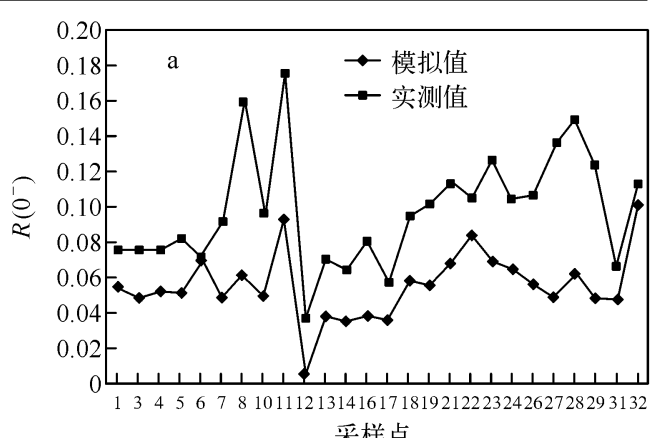

采样点
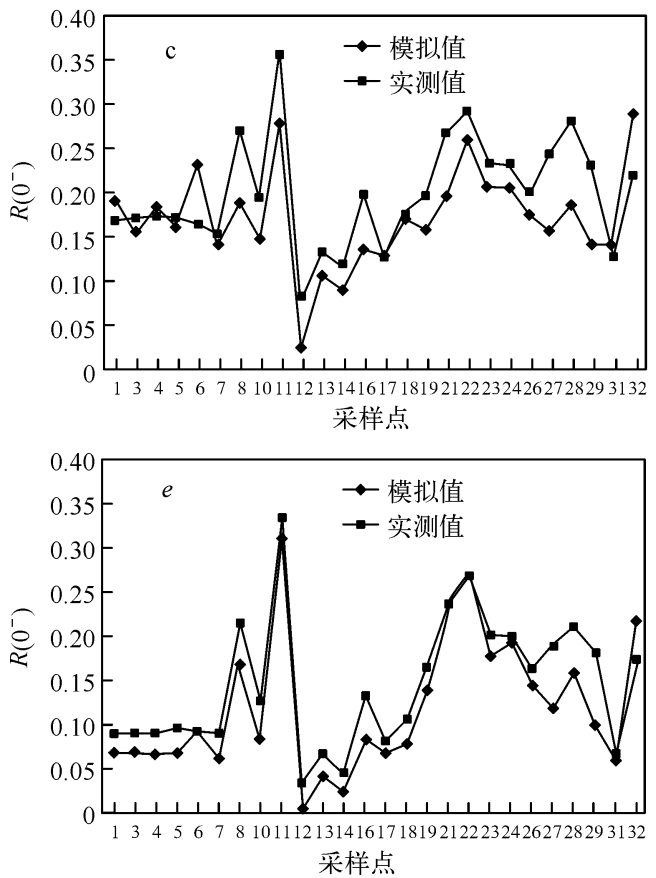

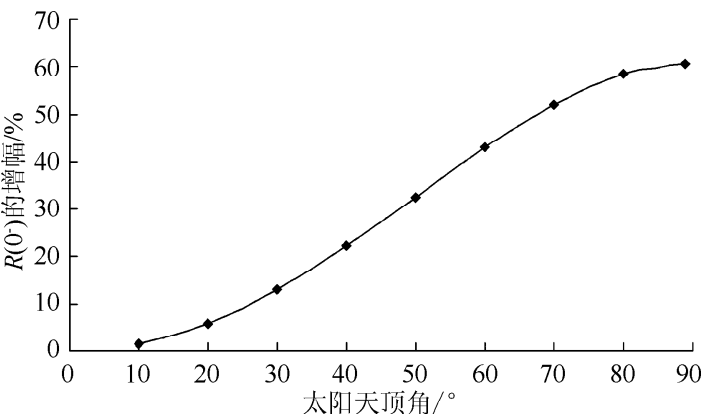

图 6 太阳天顶角变化时恰在水面下辐照度比的增幅 Fig. 6 The increase of irradiance reflectance just beneath the water surface with different sun zenith angles

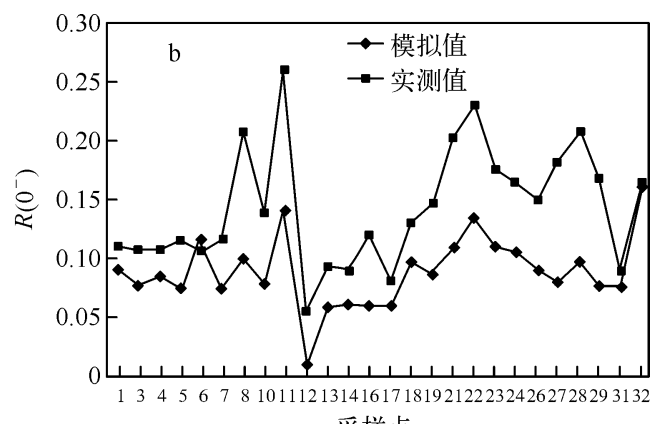

采样点

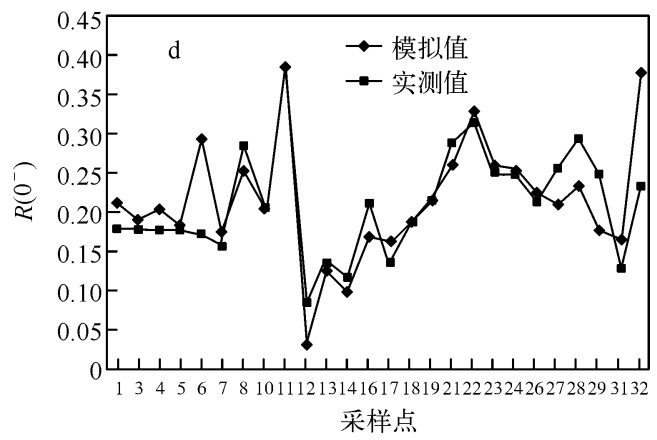

图 7 各站点实测与模拟恰在水面 下辐照度比的比较

( a: $440 \mathrm{~nm}, \mathrm{~b}: 490 \mathrm{~nm}$, c: $550 \mathrm{~nm}, \mathrm{~d}: 580 \mathrm{~nm}$, e: $676 \mathrm{~nm}$ )

Fig. 7 Comparison of measured and simulated irradiance reflectance just beneath the water surface of different stations ( a: $440 \mathrm{~nm}, \mathrm{~b}: 490 \mathrm{~nm}$, c: $550 \mathrm{~nm}, \mathrm{~d}: 580 \mathrm{~nm}$, e: $676 \mathrm{~nm}$ ) 


\section{3 讨论}

恰在水面下辐照度比主要是由水体的固有光学特性决定的, 但在一定程度上与太阳天顶角有弱相关关 系. 光束传播过程中, 进人介质时发生反射和折射. 发生折射的那部分光在经过多次散射时, 前向散射和后 向散射中都包括形成向上辐射的那部分光子 ${ }^{[5]}$. 在 Kirk 的公式中, 没有考虑体散射函数的变化, 只选择了一 个固定的 VSF,但在实际运用中, 还是会出现微小差异.

结果表明, 实际测量的恰在水面下辐照度比与模拟值在蓝紫光波段拟合度不是很高. $500 \mathrm{~nm}$ 以下是可溶 性有机物的强吸收波段, 同时叶绿素和类胡萝卜素也有一定的吸收作用. 而在实验室条件下并不能精确测量物 质的吸收、散射系数时, 当激光穿透比色血时, 必定有一部分光散射到接收器无法接收的地方, 因此测定的吸收、散 射系数有一定的误差;同时波长越短, 散射越大,因此在短波波段处, 实测的辐照度比与模拟值差异较大.

在相关性计算中, 发现 $6^{\#}$ 点和 $20^{\#}$ 点为两个异常点, 尤其是 $20^{\#}$ 点, 其趋势与模拟的结果相悖. 出现此现 象的原因可能是在进行数值模拟时, 采用的模型存在一定的缺陷. Gordon ${ }^{[4]}$ 指出, $R\left(0^{-}\right)$随着散射相函数因 子改变; Kirk ${ }^{[5]}$ 也提出, 在太阳高度角很小的时候, 恰在水面下辐照度比与 $b / a$ 也会呈非线性关系, 而本文的 数值研究都是建立在与 $b / a$ 线性关系的基础上. 另外根据 Morel 等 ${ }^{[6]}$ 的研究, 单次散射反照率也会对恰在水 面下辐照度比产生影响,而本文没有考虑.

本文数值试验都是以太湖水体水质均匀、表面平滑为前提的,没有考虑风和波浪的影响. 赵巧华等 ${ }^{[10]}$ 研究 发现, 风浪对水体表层光场的几何结构有明显的影响. 对不同人射光场而言, 不同风速对其几何结构改变程度及 趋势的影响明显不同, 天空漫射光的比例也会随之变化, 则对测量上下行辐照度带来很大误差. 太阳光照射在起伏 的水体表面,很有可能形成凸(凹)透镜, 产生聚焦(发散)作用, 因此对测量结果也会有影响. Albert 等 ${ }^{\left[{ }^{[14]}\right.}$ 也表明风 和水体表面的几何结构会对辐照度比产生影响. 因此, $6^{\#}$ 点和 $20^{\#}$ 点可能基于以上原因产生异常.

\section{4 参考文献}

[ 1 ] 戴永宁, 李素菊, 王学军. 巢湖水体的表观光学特性测量与分析. 中国环境科学, 2008, 28(11): 979-983.

[ 2 ] 马荣华, 宋庆君, 唐军武等. 太湖秋季水体遥感反射比的简单经验估测模型. 湖泊科学, 2007, 19(3).

[ 3 ] Kirk JTO. Light and photosynthesis in aquatic ecosystem. Cambridge: Cambridge University Press, 1994.

[ 4 ] Gordon HR. Dependence of the diffuse reflectance of natural waters on the sun angle. Limnology and Oceanography, 1989, 34(8) : 1484-1489.

[ 5 ] Kirk JTO. Dependence of relationship between inherent and apparent optical properties of water on solar altitude. Limnology and Oceanography, 1984, 29(2): 350-356.

[6] Morel A, Gentili B. Diffuse reflectance of oceanic waters: its dependence on Sun angle as influenced by the molecular scattering contribution. Applied Optics, 1991, 30(30): 4427-4438.

[ 7 ] Mitchell BG. Algorithms for determining the absorption coefficient for aquatic particulates using the quantitative filter technique( QFT). Proceedings of SPIE, 1990, 1302 : 137-148.

[ 8 ] Cleveland JS, Weidemann AD. Quantifying absorption by aquatic particles: A multiple scattering correction for glass-fiber filters. Limnology and Oceanography, 1993, 38: 1321-1327.

[ 9 ] Bricaud A, Morel A, Prieur L. Absorption by dissolved organic matter of the sea (yellow substance) in the UV and visible domain. Limnology and Oceanography, 1981, 26: 43-53.

[10] 赵巧华, 秦伯强. 风浪对水体表层下行光场平均余弦影响的数值研究. 水科学进展, 2006, 17(4): 531-537.

[11] Liou KN. 大气辐射导论: 第 2 版. 北京: 气象出版社, 2004: 93-118.

[12] Petzold TJ. Volume scattering functions for selected natural waters. California: Scripps Institution of Oceanography, 1972 : $72-78$.

[13] Kirk JTO. Estimation of the absorption and the scattering coefficients of natural waters by use of underwater irradiance measurements. Applied Optics, 1994, 33(15) : 3276-3278.

[14] Alber A, Mobley CD. An analytical model for subsurface irradiance and remote sensing reflectance in deep and shallow case-2 waters. Optics Express, 2003, 11(22) : 2873-2890. 